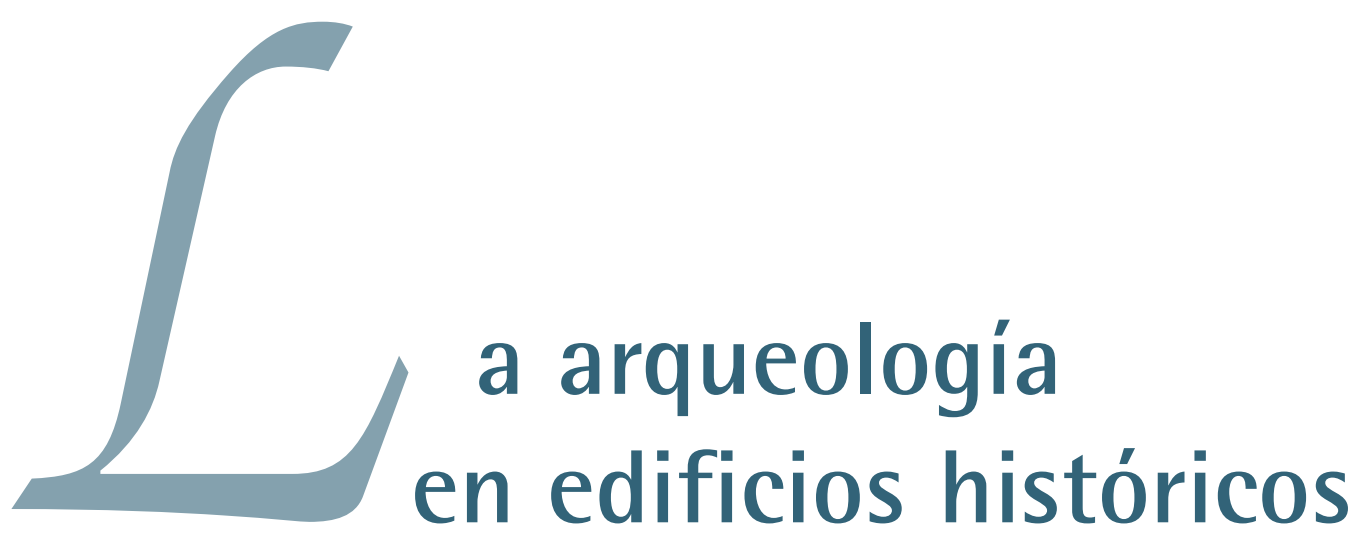

\title{
Propuesta de intervención y análisis global a través de la experiencia sevillana
}

En el mes de Diciembre de 1992, en la ciudad de Sevilla, se iniciaban las tareas arqueológicas de investigación en uno de los más emblemáticos edificios de la ciudad: el Cuartel del Carmen, antigua "Casa Grande" de la orden del Carmen en Andalucía.

Durante los años anteriores las distintas actuaciones de apoyo a la restauración o rehabilitación de inmuebles habían ido dando cuerpo en nuestra ciudad a un sistema de intervención que pretendía resolver las trabas a las que cada profesional debía hacer frente, y que se manifestaban de modo claramente insatisfactorio en los primeros trabajos. De este modo, y basándonos en los presupuestos metodológicos ya experimentados en otros lugares como Italia - Cataluña, así como en los trabajos realizados por L. Caballero y otros investigadores españoles, y sobre todo, tras un análisis autocrítico de la problemática arqueológica sevillana, surgió un primer intento experimental, materializado en el Convento de Santa María de Los Reyes de Sevilla. (I)

Tras este primer trabajo, se procedió a valorar los efectos del sistema empleado, corrigiendo y ampliando todos los aspectos metodológicos del engranaje que conformaban nuestra propuesta, de modo que, tras elegir el Cuartel del Carmen como el edificio ideal para poner en práctica lo teorizado, se pasó a aplicar de manera experimental cada uno de los elementos teóricos. (2)

Este escrito pretende divulgar de manera sintética nuestra experiencia, haciendo especial hincapié en los antecedentes locales y en las carencias que se han pretendido resolver. La validez del sistema de intervención propuesto ya ha quedado demostrada en un amplio número de edificios durante los últimos cinco años; no obstante, y de la misma forma en la que esta disciplina recien nacida, va evolucionando, no queremos dar a nuestro análisis otro sentido que no sea el de textimonio referencial de este momento metodológico. Somos conscientes que en años venideros, la arqueología y la rehabilitación irán adecuando sus esquemas aún más, ya que su relación no ha hecho más que iniciarse y aún se encuentra en período de experimentación y reflexión.

\section{CONSIDERACIONES GENERALES}

La especialización progresiva de métodos y sistemas de investigación en el campo de la arqueología del edificio ha tenido diferentes hitos e incluso distintas vías de acercamiento. En la actualidad, existen varios focos donde se han desarrollado autogenésicamente sistemas de intervención perfectamente válidos para el objetivo del conocimiento intensivo del edificio. Así, en Europa Occidental, y sobre todo en Italia y España (en el resto de Europa, a menor escala), ante la prolificación de las rehabilitaciones, han surgido focos autónomos como el encabezado por Caballero Zoreda y otros investigadores castellanos (3), el del Servicio de Conservación de la Diputación de Barcelona, (4) el de la Universidad de Granada o el de Jaén, los más famosos de Siena, Florencia, Milán, etc...y el grupo de investigadores sevillanos. (5)

Todos ellos y otros más en el resto del mundo han trabajado durante los años setenta y sobre todo los ochenta, ignorándose mutuamente debido al caracter extremadamente local de las exigües publicaciones referentes al tema. Hasta fines de la pasada década no surgieron compendios generales, fruto de las más recientes experiencias, todos ellos circunscritos al área italiana, la más prolija y sensibilizada con el tema. El resto de áreas ha generado un número muy reducido de publicaciones referentes al tema, alguno de los cuales fueron emblemáticos en su momento y pueden ser considerados pilares generales en esta disciplina: es el caso de la sistematización de Harris (6) o de la adaptación específica de Carandini (7). En cada zona se escribieron enfoques muy cercanos, los unos de los otros, referentes a las nuevas posibilidades del método arqueológico en la restauración, pero únicamente a fines de los ochen-

\section{Miguel Ángel Tabales Rodríguez}

Arqueólogo 
ta y a comienzos de los años noventa se han acometido intentos formales de sistematización de la disciplina. Nos referimos a los trabajos compilatorios de Rita Tagliabue (8), basados a su vez en trabajos anteriores de Broggiolo (9), Parenti (10), Francovich (I I), etc...

En resumen, podría decirse que el interés por el edificio histórico desde un punto de vista arqueológico y el consecuente desarrollo de la disciplina, se asentó sobre dos pilares esenciales; por un lado, el nacimiento de la arqueología urbana de urgencia y por otro, el desarrollo de la Arqueología postclásica o medieval. Ambos fenómenos fueron paralelos; los paises citados, debido a su riqueza histórica urbana y al alto nivel de vida desde los años cincuenta, dedicaron un gran esfuerzo a la renovación de sus ciudades, fruto de la cual surgió la necesidad de documentar los restos que salían a la luz. El caracter pluriestratificado de las grandes ciudades europeas motivó que, tras años de estudio de los niveles postclásicos, la disciplina arqueológica medievalista incorporara el ámbito urbano como objeto de sus indagaciones. El respeto por los edificios históricos y la necesidad de rehabilitación de gran parte de ellos, trajo consigo la aplicación de la arqueología como método indispensable para su comprensión. Esto no fue posible previamente porque, en primer lugar, no existían arqueólogos especializados en la época medieval, ni existían organismos que controlaran las obras urbanas; tampoco existía aun la figura del arqueólogo de gestión, capaz de intervenir en un lugar no programado y no se conteplaba la necesidad de conocer científicamente la evolución de un palacio, monasterio, etc... para rehabilitarlo.

La tradición arqueológica anglosajona, y su caracter pionero en los trabajos urbanos y medievalistas, originaron un clima idoneo durante los años setenta, que permitió la aparición de estudios específicos sobre edificios medievales tratados desde la óptica arqueológica. Las investigaciones de Rahtz y Hirst en la Abadía de Bordesley, Rodwell en las iglesias de Rivenhall y Hadstock en Essex, fueron algunas de las actuaciones que más influyeron en trabajos posteriores como los de Rodwell, Taylor, Butler, Rahtz, Carver, Hurst, etc

En ellos estuvo muy presente la formación teórica y metodológica de la Universidad inglesa, fruto de la cual surgieron las herramientas básicas para los futuros arqueólogos de edificios; nos referimos al método Harris (13), en lo referente a la documentación de estratos y los aportes sobre pluriestratificación presentados por Carver Martin (14). También, ya en los ochenta, se iniciaron acercamientos al análisis estratigráfico paramental como los de Hurst ( 15 ), Rahtz (16), en Inglaterra o M. Davies (17) en Australia. Ni que decir tiene que a excepción del sistema Harris, el resto de aproximaciones fueron similares y paralelas a las que se desarrollaban en el resto de Europa; sin embargo, la base teórica y metodológica fundamental para el posterior desarrollo de la disciplina surgió aquí.
En el resto de Europa destacaron durante los años setenta los trabajos realizados en Westfalia-Lippe (18), y en otras zonas de Alemania, donde se estableció la figura del Investigador del edificio (Bauforschung) como apoyo al arquitecto en el proceso rehabilitador. A nivel estratigráfico supuso un gran impulso, anterior a los trabajos de Carver Martin, el estudio sobre la problemática en los yacimientos pluriestratificados presentados por los polacos Maetzke, Rysiewska, Tabaczynski y Urbanczyk (19). En Francia, con una tradición propia de estudios arqueológicos en edificios ( Lauffray, Sanpaolesi...) (20) surgieron aproximaciones metodológicas sobre edificios concretos; es el caso de los castillos medievales, de De Bouard (2I).

No obstante, fue en Italia donde se produjo un mayor desarrollo en la dinámica de investigación y búsqueda de sistemas adecuados para la comprensión arqueológica de los edificios, asimilando los principios estratigráficos de Harris, la tradición urbana y de "urgencias", los fundamentos de la pluriestratificación, etc... y en un ambiente propicio de impulso a la rehabilitación histórica.

La figura de Andrea Carandini (22) fue una de las bazas fundamentales en este proceso, sobre todo a raiz de sus trabajos sobre la necesidad de asumción de una carta general sobre la intervención arqueológica, en 1977, y la propuesta de utilización de fichas de control para cortes, estratos y materiales. Dos años más tarde Ricardo Francovich reivindicaba el papel de la investigación arqueológica en edificios históricos (23), poniendo como ejemplo la situación alemana, y abogando, al igual que Francesco Gurrieri (24), por la coordinación "legal" con la figura del arquitecto. Este último investigador planteaba la necesidad de especializar los equipos de profesionales adscritos a una rehabilitación y la creación de vínculos osmóticos entre la Administración y la Universidad.

Con la actuación en el complejo pluriestratificado de San Silvestro, en Génova, comenzó de la mano de Bonora (25) uno de los aspectos definitorios de los posteriores estudios arqueológicos italianos: el análisis paramental estratigráfico. Al igual que en España, la primera mitad de los ochenta fue un período de especial intensidad en los trabajos de restauración del patrimonio edificado. Trabajos como los de Tiziano Mannoni (26), respecto a la datación de edificios, Fossati (27), sobre análisis matemáticos y tipológicos de medidas de materiales arquitectónicos, o Isabela Ferrando (28) sobre las seriaciones tipologicas de edificios históricos no monumentales, antecedieron a los primeros intentos serios de sistematización. En 1985, Roberto Parenti, basándose en la experiencia en la Torre A de Montarrenti (29), aportó el primer estudio sistemático de un edificio desde un punto de vista exclusivamente paramental, añadiendo tipologías murarias especialmente útiles para el medievo italiano.

Tras estos trabajos y otros, realizados fundamentalmente en el Norte de Italia, se multiplicaron las ex- 
periencias y aplicaciones arqueológicas, desatándose una polémica por parte de algunos arquitectos, encabezados por Renato Boneli (30), sobre la innecesariedad de realizar estudios arqueo-paramentales. La oposición a esta postura minoritaria la protagonizó Francovich, quien en 1988, junto con Parenti, promovió el primer compendio sobre Arqueología y Rehabilitación (3I). En él se dieron cita los mejores exponentes de la arqueología del edificio durante la década: Manacorda, Cabona, Marino, Doglioni, Broggiolo, etc... Algunos, como Manacorda y Cabona (32), transmitieron sus experiencias focalizadas desde sus áreas de trabajo en Roma y la Liguria. Otros, como Luigi Marino (33) realizaron un análisis histórico de la rehabilitación poniendo de manifiesto las carencias y problemas de las relaciones interprofesionales (comunes al resto de Europa). En este sentido, Doglioni, analizó el caso italiano de modo específico y propuso un sistema de estudio de los paramentos basados, antes que en los aspectos estratigráficos y cronológicos, en las caracrésticas de la fábrica y sus componentes. (34)

Roberto Parenti analiza las relaciones estratigráficas, comentando los distintos tipos de registros utilizados hasta el momento; transforma parcialmente la relación de coetáneidad de Harris, realiza estudios tipológicos, analiza los indicadores cronológicos siguiendo a Manacorda, estudia los materiales, aparejos, dimensiones, etc...,(35) creando así un conjunto de sistemas enfocados al conocimiento exhaustivo de las estructuras visibles del edificio. El contrapunto, por lo que se refiere al subsuelo, lo pone Maura Medri (36) con su desarrolllo de las plantas compuestas en excavación.

Junto a Parenti, en lo que se refiere a los estudios paramentales, Gian Pietro Broggiolo, asumió en la segunda mitad de los años ochenta un papel esencial, tras la realización de una serie de estudios destinados a la sistematización de los análisis murarios. Destaca entre todas sus aportaciones la incorporación de la jerarquización de las unidades estratigráficas según su disposición referencial, así como las gradaciones de las necesidades en los muestreos y su cumplimentación en fichas oportunas. Los trabajos de Broggiolo culminaron en 1988 con la edición de un manual sobre técnicas de investigación edilicia, (37) donde se desarrollan, desde un enfoque estrictamente arqueológico, cada una de las propuestas anteriores, añadiendo una disertación teórica sobre las acciones de la rehabilitación y la incidencia de la intervención arqueológica en el marco de las actividades interdisciplinares que la conforman.

Ya en los años noventa, la arquitecta Rita Tagliabue (38), desde la Universidad Politécnica de Milán, acometió el intento más completo (realizado hasta el momento) de organización de todos los factores que intervienen en el proceso de la investigación previa a la rehabilitación, desde un enfoque arquitectónico, pero con un grado de profundidad teórica nada desdeñable.
Ante todo, se enfrentó frontalmente al verdadero problema de fondo en este tipo de actuaciones, esto es, las relaciones interdisciplinares, sobre todo la relación entre investigador y diseñador (o arqueólogo-arquitecto). Sintetizando todos los aportes previos, llevó a cabo una recopilación muy compleja, basándose sobre todo en Parenti, Broggiolo, Mannoni y Bonelli. De este estudio se desprenden múltiples interpretaciones, pero las dos principales son, a nuestro entender, por un lado, la asumción absoluta por parte de los arquitectos-rehabilitadores de la necesidad de una investigación arqueológica completa del edificio, y por otra, la urgencia en la formación de los arquitectos en el método arqueológico, de modo que sean éstos (ayudados por los arqueólogos) los que acometan los estudios paramentales.

En los últimos años, y como fruto del nuevo ambiente cultural europeo, se han multiplicado las experiencias arqueológicas en todos los ámbitos relacionados con el patrimonio edificado; no hay mes en el que no se organicen jornadas, seminarios, congresos, etc... sobre nuestro Patrimonio Histórico y su conservación. Dentro de esta "vorágine", la interpretación arqueológica cobra cada vez un lugar más lógico para ese fin. En este sentido, nuestro análisis pretende ser una pequeña pieza más en ese rompecabezas primigenio, que sin duda culminará por completarse en las próximas décadas, una vez que los estudios histórico-arqueológicos previos terminen su período reflexivo y práctico, y acabe por aceptarse una fórmula equilibrada, hoy aún muy lejos, entre la investigación y la creación arquitectónica (39).

\section{LA EXPERIENCIA SEVILLANA}

El panorama de la Sevilla de los años ochenta, en lo que a arqueología aplicada a la rehabilitación respecta, no es especialmente intenso, si se tiene como referencia la actividad desarrollada en ese período en el resto de Europa o en otras zonas de España. Figuras como la de Alfonso jiménez, representante de una tradición de historiadores-arquitectos en la que se encuadran nombres como los de R. Manzano, Félix Hernández, etc...., representan un foco prolijo de actividad práctica y teórica que mantiene no obstante una altísimo nivel en lo que se refiere al interés por el conocimiento integral del edificio antes de su restauración.

En 1987 y 1988 se celebran el I y II Curso del COAAO (40) sobre Rehabilitación y Ciudad Histórica, y sobre Restauración y Análisis arquitectónico; en dicho foro, y mientras en Siena o Florencia surgen trabajos "recopilatorios" sobre métodos y sistemas de trabajo aplicados al conocimiento histórico y constructivo de los edificios en rehabilitación (4I), durante la última década, aquí se suceden las reflexiones teóricas más dispares y se exponen los últimos trabajos en la ciudad sin mencionar una sola palabra de Arqueología, salvo en algún caso, y de manera muy anecdótica. 
Durante el año 1985, se producirá un cambio sustancial en nuestra ciudad, con la entrada en funcionamiento del equipo arqueológico de la Delegación Provincial de Cultura de la Junta de Andalucía, a cuya cabeza operarán, primero F. Amores (42), y luego J. M. Campos (43).

En esos momentos se interviene desde la administración en algunos edificios con procesos de rehabilitación ya iniciados, o con vistas a su protección, por lo general, para su inclusión en el Plan General de Ordenación Urbana de Sevilla, en redacción por entonces; es el caso del Cortijo de Miraflores (44) o la Buhayra,(45) dirigidos por F. Amores, o los Baños de la Reina Mora y las Murallas de la Macarena, a cargo de J.M. Campos y J. Escudero (46).

En el año 1986, las actuaciones de apoyo a la restauración se disparan. Según los arqueólogos provinciales, este tipo de intervenciones suponen por entonces un índice objetivo del cambio de actitud que la arqueología de gestión está logrando. En esos momentos se interviene en el Antiguo Noviciado de San Luis de los Franceses (47)aplicándose el método arqueológico para determinar la evolución arquitectónica de los sectores más confusos del edificio, previo a su restauración; para ello, se realizaron excavaciones donde se obtuvieron los vestigios de una villa y una posterior alquería, tomándose datos de los alzados. En la misma tónica, se iniciaron las excavaciones en el Convento de San Agustín, en la Cartuja, en las murallas de los Jardines del Valle y en la Casa de la Moneda. (48)

En los dos primeros conventos se iniciaron las tareas de documentación y excavación parcial de algunas de las zonas más conflictivas, con vistas a la restauración de ambos monumentos. Los objetivos, planteados a largo plazo, motivaron que los trabajos fueran acometidos de manera sectorial, con absoluta dedicación al subsuelo. En la Cartuja se analizaron las capillas de Sta Magdalena y las de las Santas Justa y Rufina, continuándose los trabajos en otras áreas hasta el año 1992. Más adelante analizaremos la evolución metodológica observable en la Cartuja durante ese período, así como su contribución fundamental al proceso del que tratamos.

Tanto las murallas del Valle como la Casa de la Moneda fueron proyectos promovidos por la Gerencia Municipal de Urbanismo del Ayuntamiento sevillano. En estos y en otros trabajos futuros como los del monasterio de San Jerónimo se aplicaron las más diversas técnicas para la solución de problemas de cotas y cronología, y en el caso del segundo edificio, para completar la detección del amurallamiento almohade.

En todos los casos, se trata de incursiones en monumentos o edificios históricos en proceso de restauración o rehabilitación, efectuadas bajo unos presupuestos metodológicos propios de la recién creada "arqueología de gestión", imbuida sobre todo de urbanismo y evolución urbanística. De hecho, se exca- va el subsuelo del inmueble como si de un solar más se tratara. Las observaciones sobre la estructura emergente son más propias de la visión de un historiador del arte que de un arqueólogo; es decir, se excava de la manera más rigurosa posible para localizar piezas que faltan en el engranaje evolutivo pero sólo complementando la información respecto a lo paramental con análisis muy generales.

Se evidencia una procedencia formacional clara de los investigadores-arqueólogos que reflejan conductas propias de las excavaciones sistemáticas en las que la universidad sevillana, tan alejada de la problemática arqueológica urbana y postclásica, se desenvolvía.

El valor de este momento es innegable, pero no debemos olvidar que por entonces, por poner un ejemplo, en la Diputación de Barcelona operaba un Servicio de Intervención en el Patrimonio Edificado, con arqueólogos, historiadores, arquitectos, etc.. que ya había publicado cuatro compendios de experiencias arqueológicas de apoyo integral a la rehabilitación en inmuebles, desde 1979; por otro lado, C. Zoreda ya había publicado sus experiencias en varios trabajos metodológicos, y el Compendio de 1988 sobre Arqueología y Restauración de Monumentos de Florencia ya había trascendido.

En Sevilla, sin embargo, continuábamos sin aplicar ni siquiera el método Harris, y las lecturas paramentales eran algo impensable. En los años siguientes todo cambiaría rápidamente, tal y como ahora veremos, sobre todo gracias a dos pilares fundamentales, además del ya comentado de los trabajos a cargo del equipo técnico de la Delegación provincial de Cultura, en colaboración con la Gerencia Municipal de Urbanismo.

Nos referimos por un lado a la evolución de los trabajos arqueológicos en la Cartuja de Santa María de las Cuevas, a cargo de F. Amores, y por otro a la labor de D. Oliva, conservador del Museo Arqueológico Provincial, en los Palacios de Altamira y de Miguel de Mañara en el barrio de San Bartolomé.

Una de las fechas importantes en nuestra ciudad es la de 1986, momento en el que se inician los primeros trabajos globales en la Cartuja.

Entre los años 1987 y 1989 se mantuvieron las intervenciones en la Cartuja (49) y en el Noviciado de San Luis (50), iniciándose los estudios de los Palacios de Altamira (5I) y Mañara (52), así como los Monasterios de San Clemente (53) o San Jerónimo. (54) También se realizaron estudios arqueológicos en la Torre de la Plata, como culminación de la investigación global y emblemática de la Gerencia Municipal de Urbanismo en el sector de la Moneda. (55)

En la cartuja, en 1987, se amplió la excavación desde las capillas al Claustro de Monjes, liberándose por completo y sirviendo de pruebas para una interesante experiencia de divulgación consistente en el fotografiado aéreo de las estructuras excavadas, tra- 
tadas cromáticamente en función de su adscripción cronológica. Durante los años siguientes, las campañas se extendieron y diversificaron a lo ancho del conjunto monacal y de la posterior fábrica de cerámica, destacando los estudios de las huertas, la capiIla de "Afuera", los distintos claustros, la iglesia, etc...ultimándose en 1991 un ingente esfuerzo de documentación dificilmente superable en el futuro, que culminó con la total rehabilitación en 1992.

Fueron años en los que se pasó en la Cartuja, desde un estadio inicial de estudios parciales, durante las primeras campañas, hasta una situación de investigación global y generalizada, a la par que científicamente innovadora.

En el Noviciado de San Luis, los trabajos realizados en las campañas de 1987 y 1988 , sirvieron de manera muy puntual a los propósitos de la rehabilitación, aportándose datos sobre bóvedas subterráneas de los siglos XVII y XVIII, y de estructuras romanas e islámicas. En ningún momento se pudo optar por un análisis integral debido al ritmo de obra al que se supeditaban las actuaciones arqueológicas. Este mismo problema condicionó los trabajos en San Clemente, durante 1989 y las primeras investigaciones en el Palacio de Mañara. En el primero, la investigación se centró en la actual iglesia (s. XVI) y en una estructura subterránea con restos islámicos ubicado al pie de la estructura primitiva del cenobio. En el segundo, se pudieron realizar cortes dispersos pero muy restringidos. En ambos casos, sin embargo, fue posible ampliar la misión de la investigación desde ésas primeras tomas de contacto, hasta verdaderos estudios integrales (no superados hasta el momento en nuestra ciudad), gracias al arquitecto Fernando Villanueva Sandino, director de ambas rehabilitaciones y a arqueólogos como Diego Oliva Alonso.

Este investigador desempeñó junto a otros arqueólogos como E. Larrey, Sandra Rodríguez, R. Ojeda y M.A. Tabales, un papel determinante en nuestra ciudad, asumiendo una vía de análisis multidisciplinar, siempre con caracter integral, que desembocaría a partir de 1990, en la creación de nuestro sistema de trabajo, aplicado ya en otros monumentos posteriores.

En el Palacio de Altamira, durante los años 1988 y 1989 se analizaron todas las dependencias y se relacionaron todas las estructuras emergentes con las distintas fases medievales, arrancando la investigación desde fases romanas. Junto a la excavación, destacaron los estudios documentales, los análisis de materiales, etc..

En el monasterio de San Jerónimo se actuó también de manera integral, pero con la salvedad de una carencia de medios absoluta y una imposibilidad de asumir desde la arqueología, todos los frentes de la investigación histórica de apoyo a la rehabilitación, debido al exigüe papel que por entonces la Gerencia de Urbanismo otorgaba a nuestra disciplina dentro de la investigación en edificios. No obstante, y debido al caracter bifásico del sustrato, y a la poca potencia de relleno, se pudieron resolver la mayoría de incógnitas planteadas.

Según nuestro modo de ver, y durante el período que va de 1985 a 1993, el conjunto de las actuaciones citadas, puede agruparse desde varios puntos de vista.

- El primero, es el que distingue a aquellas investigaciones de Apoyo a la Restauración integradas dentro del proyecto arquitectónico, y valoradas de manera global, y generalmente previa a la fase de obras. Es el caso menos común en esos momentos, y se restringe a dos trabajos emblemáticos que definieron dos vás de interveción diferente durante los años siguientes; se trata de las macrointervenciones en la Cartuja de Santa María de las Cuevas, y la del Palacio de Altamira. El resto, son trabajos más o menos amplios pero generalmente parciales en cuanto a sus objetivos, y por lo general insertados ya durante el proceso de obras de rehabilitación.

- Desde el punto de vista de la utilización más o menos avanzada de métodos arqueológicos específicos para investigaciones de este tipo, y asumiendo también lógicamente, los contenidos del punto anterior, podemos asumir una clasificación algo más compleja, pero mucho más lógica desde la concepción de éste trabajo. Observamos pués los siguientes tipos-estadios de actuaciones:

I. Trabajos del tipo I. Se caracterizan por ser investigaciones parciales de edificios, generalmente condicionadas por su inserción a posteriori en los procesos de obra de rehabilitación, y realizadas por lo general con una visión aún muy parcial de los estudios sobre el patrimonio edificado. Comúnmente, consisten en meras excavaciones realizadas "en el edificio", pero no necesariamente con éste en el primer orden de prioridaddes (aunque después se intente completar la falta de atención a la estructura emergente con análisis por lo general superficiales y de carácter documental o artístico, del conjunto del monumento en cuestión). Metodológicamente son realizadas cada una según la formación del investigador y sus afinidades y generalmente no siguen el sistema de distinción de unidades estratigráficas. A este primer grupo, que coincide con nuestros primeras excavaciones en la ciudad de Sevilla pertenecen las rehabilitaciones del Noviciado de San Luis, El Convento de San Agustín, Miraflores, San Jerónimo, etc.... A este grupo se adscribirán en los años siguientes otros trabajos como los del Monasterio de San Agustín, etc...pero por entonces (19901994) nuestro sistema de trabajo específico (que seguidamente veremos en el tipo 3, ya estaba siendo experimentado).

2. Trabajos del tipo 2. Caracterizados por su intención de análisis integral, con aspiraciones de asumir desde la óptica arqueológica el resto de los estudios multidisciplinares, generalmente bien in- 
tegrados o aceptablemente insertados en el proyecto de rehabilitación. Son los estudios de la Cartuja y del Palacio de Altamira. A este grupo se pueden (56) añadir en los años siguientes los del Palacio de Mañara, el Cuartel del Carmen y el Monasterio de San Clemente, etc...(57)

3. Actuaciones del tipo 3. Enraizadas en el tipo 2, en cuanto a objetivos generales, se distingue de aquella por la utilización de un sistema de trabajo autónomo y sobre todo específico, basado en las experiencias anteriores y destinado a suplir las carencias detectadas previamente, mediante la especialización del equipo de investigación básico y la definición de aquellos aspectos esenciales para la caracterización global del inmueble. Metodológicamente, como más adelante desarrollaremos, este grupo se caracterizó por su caracter experimental, poniéndose en práctica sistemas de registro diversos, indagándose en los estudios paramentales hasta cotas no superadas ni en nuestro entorno ni en el resto de Europa, etc. Pertenecen al grupo los trabajos en el Convento de Santa María de los Reyes, el Cuartel del Carmen, el Palacio de los Marqueses de Marchelina y con posterioridad, las actuaciones en la Puerta de Córdoba de la Ciudad de Carmona, la Iglesia de Santa Ana de Guadalcanal, etc...

\section{PROPUESTA DE INTERVENCIÓN}

Nuestra propuesta de análisis surge en 1990, tras haber experimentado en varios edificios sevillanos desde mediados de la década de los ochenta, aplicando la metodología aprendida en intervenciones arqueológicas de urgencia o sistemáticas y centrándonos casi exclusivamente en el análisis del subsuelo. Nuestro equipo participó o dirigió los trabajos realizados en el Monasterio de San Jerónimo de Buenavista, (Pozo y Tabales, 1988), el Castillo de Alcalá de Guadaira, (Tabales Y Pozo, 1989) (58), etc...

También realizó trabajos algo más avanzados, en el Palacio de Altamira ( Larrey, E. y Oliva, D., 1988), y sobre todo en el Palacio de Mañara (Oliva, Ojeda, Tabales, 1990) (59). En ellos ya estaban latentes los problemas de inoperatividad que debían resolverse imperiosamente, además de comenzar a darse algunos pasos capitales en aspectos tan esenciales en los años siguientes, como el establecimiento de grupos coherentes de trabajo, o los primeros estudios paramentales realizados en Sevilla.

En el Palacio de Mañara (1990), se pone en práctica, a diferencia que en el de Altamira o en la Cartuja, todo un programa de estudio de alzados, desde el punto de vista estratigráfico, centrado en la estancia de las pinturas mudéjares, caracterizado por el uso rígido del sistema Harris, ya aplicado puntualmente en el Palacio de Altamira. Además se levanta con criterios arqueológicos todo el sector Suroriental de la casa, previamente a su derrumbe.
Los resultados son buenos pero representan una anécdota en la investigación global y más que un aporte esencial en nuestro trabajo, supusieron una experiencia "interesante" aunque lejana en cuanto a operatividad, sobre todo, por la carencia de una estrategia clara.

No debemos olvidar "ni ocultar" la ausencia, en aquellas fechas, de contacto y el desconocimiento de los trabajos de otros profesionales en este campo, tanto en España (básicamente Caballero Zoreda, en Castilla y López Mullor para la Diputación en Barcelona), como en Italia y otros paises desde los finales de la década anterior. Nos consta que hasta finales de los años ochenta no comenzaron a divulgarse los trabajos de este tipo entre los profesionales de distintas zonas. En este caso, fue en esos momentos cuando entramos en contacto con los citados autores así como con los clásicos trabajos sobre estratigrafía muraria de Parenti, Francovich, Broggiolo,etc...

A finales de 1990, los trabajos realizados por nuestro equipo en el Cuartel del Carmen (Tabales, M.A. 1990) y el Monasterio de San Clemente (Tabales, 1991) (60), ofrecieron la posibilidad de aplicar un embriónico "sistema de actuación", completamente propio en cuanto que se fundamentaba en la observación y superación de las carencias evidenciadas en los edificios anteriores. (por aquel entonces, nuestra presencia en la mayoría de ellos nos daba cierta perspectiva, al menos de lo que debería asumirse y generalmente se eludía por falta de tiempo o dinero).

El sitema en cuestión se basaba en la aplicación de un cuerpo de fichas de registro, (unidades estratigráficas y seguimiento arqueológico); además, el estudio de los paramentos y techumbres asumía ya un papel primario y no anecdótico. Por último, se intentaba obtener un equipo prioritario de trabajo a la par que especulábamos sobre las fases de actuación lógicas en cada edificio.

Por lo que respecta al sistema de registro, optamos en principio por seguir el modelo de fichas de unidades creado por R. Lineros para la ciudad de Carmona (1986); así, en el Carmen 90 y en San Clemente 91. En cuanto a el resto de fichas, de seguimiento de obras y de control de bienes muebles, se utilizaron fichas propias basadas en las utilizadas en los Palacios de Altamira y Mañara, diseñadas por D. Oliva (1988).

En general, los resultados fueron positivos, sin embargo, era obvia una clara disfunción entre los medios de los que disponíamos y el grado de exigencia al que sometía la cumplimentación de un registro de esas características.

El sistema de actuación diseñado tras la ultimación de la Rehabilitación del Convento de Santa María de los Reyes (1992) (61) fue puesto seguidamente en práctica a gran escala en El Carmen caracterizándose por los siguientes preceptos: 


\section{En cuanto a la estrategia global de intervención:}

a. Acercamiento inicial al edificio.

- División zonal, identificación de "unidades-guía"y estancias.

- estudio previo y pormenorizado de la estructura emergente.

- estudio de los sistemas de adosamiento y contacto entre alineaciones.

- análisis edilicio provisional (constatación de los tipos de muro).

- realización de un programa de Muestreos edilicios.

- Cumplimentación de las observaciones tipológicas.

- Registros de elementos artísticos o susceptibles de conservación.

- Elaboración de una hipótesis evolutiva inicial.

b. Análisis de paramentos.

- Estudios estratigráficos.

- Análisis tipológicos.

- Constatación de los principales eventos estructurales.

c. Estudio del subsuelo.

- Realización de cortes estratigráficos.

- Apertura de cortes y zanjas-guía.

- Realización de cortes con caracter "extensivo"

d. El Control de Obras.

- Control de las actividades de restauración.

- Control de las obras de rehabilitación.

\section{Respecto al registro y el método.}

a. Seguimiento del método Harris con ciertas puntualizaciones.

b. Establecimiento de unos criterios de representación gráfica esenciales, tanto en las plantas y perfiles como en los alzados de tipo estratigráfico y tipológico-estructurales.

c. Cumplimentación ordenada de registros de control:

- Unidades estratigráficas.

- Bolsas de materiales.

- Registro de cortes.

- Registro gráfico.

- Registro fotográfico.

c. Utilización de un sistema de fichas apropiado:

- Ficha de unidades única para cualquier tipo de elemento (emergente o soterrado).

- Ficha de control de obras.

- Ficha de control tipológico.

- Ficha de Muestreo edilicio.

- Ficha de estado previo.
3. Respecto al equipo básico y la relación interprofesional.

a. Selección de los miembros esenciales del equipo y sus actividades.

b. Los estudios multidisciplinares. Selección y valoración.

c. La priorización de los trabajos. Condicionantes habituales.

La estrategia global que debe regir este tipo de estudios parte de la asumción de una serie de principios esenciales:

- Vinculación esencial entre estudio histórico arqueológico y rehabilitación.

- Análisis generalizado (no parcial) del inmueble.

- Comprensión eminentemente diacrónica del edificio.

- Inserción geohistórica y tipológica en su entorno.

- Sistema de acercamiento ágil pero sistemático.

- Especial atención a lo paramental.

- Análisis necesariamente selectivo primando lo general sobre lo anecdótico.

- Mantenimiento de la investigación mientras existan remociones o alteración de lo preexistente.

- Dirección del arqueólogo director de la intervención sobre todo tipo de estudios multidisciplinares: histórico documentales, artísticos, analíticos etc.. que redunden en la comprensión histórica final.

- En la medida de lo posible. capacidad al menos de opinión, en la posterior rehabilitación.

Consideramos imprescindible un tipo de acercamiento al edificio adecuado a las posibilidades económicas de la intervención; para ello no hay recetas mágicas ya que la complejidad puede esconderse tras los aspectos más insospechados. Sin embargo sí existen algunas opciones que nos ayudarán a plantear los trabajos correctamente. Nos referimos en primer lugar a la posibilidad de realizar una fase de estudios previos muy general, tal y como planteábamos en nuestra tesis de Licenciatura. Si esto no es posible, sí aconsejamos dedicar unos días a la realización de los estudios previos esenciales, tras lo cual sí estaremos en condiciones de valorar el tiempo y equipo necesarios para la intervención.

Primeramente procederemos a obtener un fichero completo en el que se reflejará el estado previo del edificio antes de la rehabilitación. Se realizará estancia por estancia, centrando nuestra atención en solerías, muros, vanos, detalles decorativos, etc..

Paralelamente, se identificarán numéricamente los ámbitos o estancias y daremos numeración a los "Paramentos Guía", o lo que es igual, a las alineaciones principales que conforman las crujías y compartimentos más destacados. Serán las primeras unidades estratigráficas de la lista que luego continuaremos. Podríamos optar por una jerarquización de las unidades tal y como propone Broggiolo 


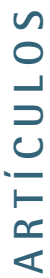

1. Sistemas de Adosamiento. Ejemplo: Intervención en el Palacio de los Marqueses de Marchelina,

2. (sup.) Distribución de Aparejos. Ejemplo: Iglesia de Santa Ana de Guadalcanal, 1996.

3. (sup.) Hipótesis Evolutiva Provisional. Ejemplo: Iglesia de Santa Ana de Guadalcanal, 1996.
(1988), pero pensamos que este tipo de disquisiciones nos alejan más que ayudarnos en el objetivo general de la comprensión global del inmueble. De hecho, si establecemos la separación entre unidades guía y unidades estratigráficas, sólo lo hacemos para poder obtener una hipótesis evolutiva inicial que nos sirva de punto de partida, y centrarnos con suficientes elementos de juicio en el estudio general posterior.

Tras esta identificación, se procede al análisis de los distintos "tipos de adosamiento"; estos pueden ser de distinto tipo: simple, con encastres simples o complejos, coetáneos, etc...Para ello utilizamos una simbología ideada para tal fin y compuesta por tres signos esenciales acompañados por una flecha que indica el orden de construcción, es decir, el apoyo o la yuxtaposición

- Paramentos coetáneos.

- Encastrados.

- Adosamiento simple.

Mediante la aplicación de estos tres tipos resumidos de contacto entre paramentos-guía de manera sistemática y global, o sea, contacto tras contacto y estancia tras estancia, obtendremos una primera planta con una lectura general clara. Por lo general, el orden de los adosamientos y su tipología suele definir con muy pocos cambios los procesos constructivos del palimpsesto.

La comprensión del modo en que contactan cada una de las alineaciones principales que configuran el esqueleto del inmueble, es indispensable para empezar a tener un dictamen sistemático de su evolución. Partimos de la evidencia, en la mayoría de los casos, de pluriestratificación y de reformas ornamentales desconexas de los procesos constructivos, lo cual su-

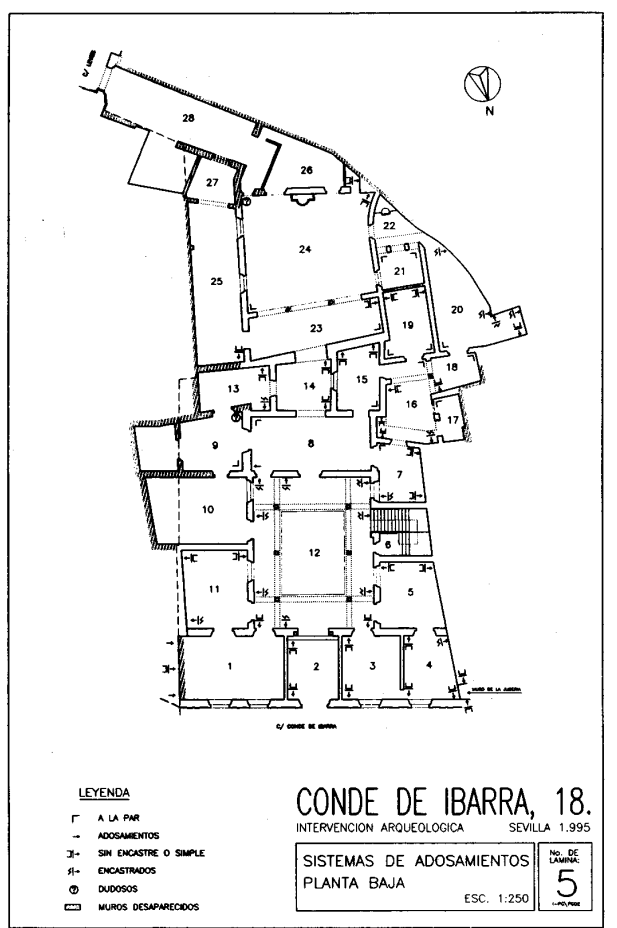

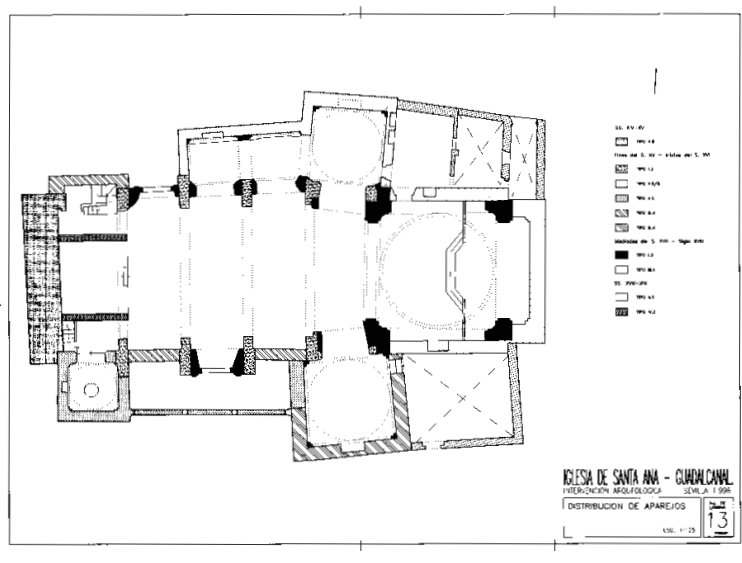

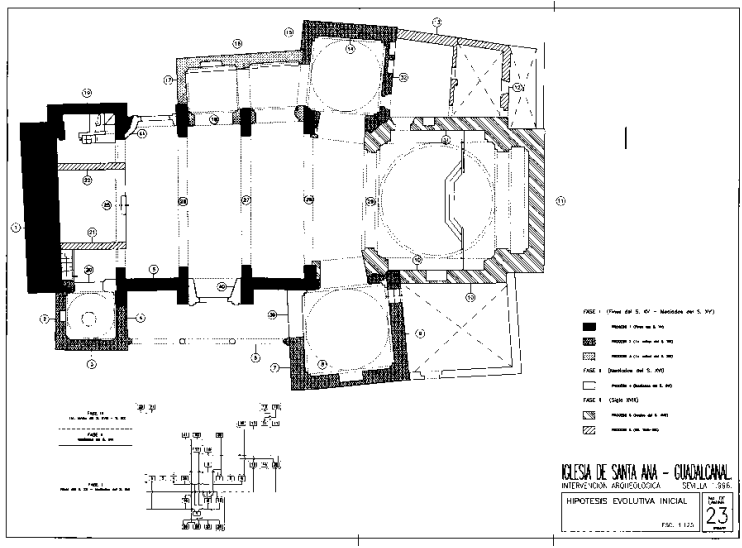

pone desde el inicio del trabajo una desvinculación de las noticias de caracter histórico o las generalmente superficiales interpretaciones artísticas.

Para poder realizar esta lectura inicial debemos acceder a la fábrica de los muros en las zonas de unión. En el caso de que existan obras de arte, pinturas o impedimentos de cualquier tipo para poder descarnar y llaguear el muro, deberemos acudir a la excavación o a la interpretación de los contactos en la techumbre, y si ésto tampoco fuera posible, recurrir en última instancia a la habitual reflexión analógica.

Sin embargo, y siempre que sea posible deben iniciarse los trabajos con el llagueado de al menos un metro cuadrado continuado, o varios puntos separados; no olvidemos que en muros pluriestratificados, como es el caso de gran parte de los edificios históricos sevillanos, se da el caso de superposiciones continuas que contactan evidentemente de manera diferente con el paramento contiguo. Por ello, como objetivo sistemático, siempre que las posibilidades económicas lo contemplen, debe aspirse a un picado arqueológico integral de la estructura.

Este picado debe realizarse, por supuesto posteriormente a un programa de catas en los enlucidos que descarte cualquier posibilidad de destrucción de pinturas o cualquier otro elemento paramental de interés artístico o arqueológico.

Distinguimos interés artístico del arqueológico ya que éste último valora principalmente los aspectos 
referentes a la interpretación evolutiva de la estructura; es decir, en un muro de varias capas de pinturas murales superpuestas (p. e. islámicas bajo mudéjares, bajo barrocas, bajo actuales), la capa pictórica puede considerarse poco importante desde el punto de vista artístico si la pintura ha desaparecido, pero la disposición de su intónaco puede resultar esencial en la lectura estratigráfica. Por ello, esa operación debe llevarse a cabo por un restaurador junto al arqueólogo director de la investigación.

Sólo tras el dictamen especializado de los expertos podrá libremente, y siempre expuestos a cualquier sorpresa, procederse al picado y llagueado de los muros.

Posteriormente se identifican las divisiones edilicias generales; es decir, se procede a rellenar sobre una planta con las unidades paramentales guía, aquellos componentes fundamentales del muro: tapiales, ladrillo, piedra, mixtos, etc..., caracterizando cada uno con una trama distinta.

Con ambos estudios, el de adosamientos y ésta identificación de fábricas esenciales, ya pueden establecerse las primeras hipótesis de trabajo. Éstas, deberán plasmarse en una planta secuenciada en la que se otorgue a cada fase constructiva una trama distinta, acompañada de una matriz Harris interpretativa, con la secuencia cronológica provisional.

Todas las actividades expuestas hasta ahora son factibles a lo largo de los primeros días de trabajo, o incluso para el caso de edificaciones muy complejas, a lo largo de la primera semana. Tras ese período ya dispondremos de una primera hipótesis de partida, y además habremos valorado cronológica y económicamente el proceso restante. En la actualidad, no es necesaria una realización de Proyecto para proceder a un análisis paramental previo como el expuesto; sin embargo sí lo es para realizar cualquier tipo de excavación, por lo que a no ser que exista una intervención preliminar en la que se ejecuten los cortes-guía, no será posible establecer la potencia del terreno ni comprobar cuales son las fases previas, antes de la obtención del permiso de excavaciones. Por tanto, y a no ser que cambien las leyes, en lo referente a excavaciones, nuestra investigación comenzará a ese respecto con unas previsiones de actuación muy generales.

Las actuaciones que ahora siguen deberán formar parte de la investigación propiamente dicha y por tanto, estarán sujetas ya a todo tipo de consideraciones estratégicas vinculadas a lo observado en el estudio previo. Es decir, ya se sabrá qué muros son prioritarios y cuales no para analizar, en cuáles se realizarán muestreos, qué zonas deberán centrar nuestra atención, etc... El objetivo es claro: sea cual fuere el resultado final, no debemos gastar esfuerzo ni tiempo en aspectos tangenciales, por muy interesantes que pudieran aparecer ante nuestros ojos.

Es ahora el momento de dedicar el máximo esfuerzo al control arqueológico y a los catálogos citados:
- Fichero artístico, en el que se reflejarán todos aquellos detalles dignos de consolidación o al menos de documentación, por su interés estilístico.

- Fichero de seguimiento de obras. En este fichero se plasmará paso por paso, cada momento de la rehabilitación, al margen de la investigación, con el fin de sistematizar de este modo todo lo ocurrido en el solar desde sus primeras ocupaciones hasta la culminación de las obras actuales.

- Ficheros arqueológicos, en los que se reflejarán todas las actividades paramentales y del subsuelo con interés arqueológico al margen de la excavación o del análisis mural: serían dos: Fichero tipológico y fichero de muestreo edilicio.

Para los dos primeros se empleará el mismo tipo de ficha, diseñada por nosotros, en la que se establece de manera gráfica y fotográfica la disposición del objeto referido y sobre todo su ubicación, mediante un plano incorporado del inmueble. En el cuestionario simple de este registro, aparte de su identificación estratigráfica, hay apartados referentes a la materia y al tipo de actividad en la que ha salido a la luz. Inicialmente (Cuartel del Carmen, 1990, y San Clemente 9I) diseñamos fichas de catalogación y control para cada tipo de elemento: madera, herrajes, azulejería, escudos, etc...pero, en realidad, la mayoría de los datos fichables eran los mismos, variando sólo los aspectos tipológicos que no tienen necesariamente que constar en fichas de control rápidas; el resultado fue la apertura de todo tipo de archivos pero con el mismo tipo de ficha de control. A este respecto, nuestra argumentación tiene la misma justificación que para nuestra ficha estratigráfica; se trata de asumir rápidamente la mayor parte de la información esencial. Huelga decir que con medios abundantes y sin condicionantes de ningún tipo, consideraciones de este tipo sobran; en ese caso mientras más y más complejas (en cuanto a captación máxima de información) sean las fichas, mejor.

Respecto a los otros ficheros, el de muestreos y el tipológico, el uso de fichas específicas se hace imprescindible debido a varias razones. Necesitan de una gama de datos estratigráficos que podrían bien asumirse en las fichas de unidades, pero por contra están sujetos a clasificaciones muy distintas, no vinculadas a la estratificación y sí a cuestiones de índole tipológica. Esto nos aconseja un uso de registros diferenciados. En el de Muestreos Edilicios, se ficha cada tipo distinto de aparejo, analizando a fondo sus elementos y relacionándolos estratigráficamente con otras unidades adyacentes, adjuntando número de muestra, medidas medias de los distintos elementos configurantes, esquemas de ubicación de la muestra, etc...

Respecto al Fichero tipológico, con la intención de completar las tipologías edilicias, se fichan por tipos cada vano, arco, suelo, cimiento, aparejo, etc...enfatizando las cuestiones métricas y en general todos los paralelos y cronologías asociadas en otros edificios locales; se completa con un apartado gráfico y otro fotográfico. 


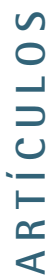

4. (sup.) Propuesta de Simbología Estructural. Tabales, M.A. 1993.

5. (inf.) Tipologías Edilicias. Ejemplo de Tipología sevillana. Tipos formales de tapial. Tabales, M.A. 1994.

6. (dcha.) Tipología sevillana de vanos (dinteles y roscas). Tabales, M.A. 1993.

7. Análisis tipológico de aparejos Ejemplo: Intervención en el Palacio de los Marqueses de Marchelina, 1995.

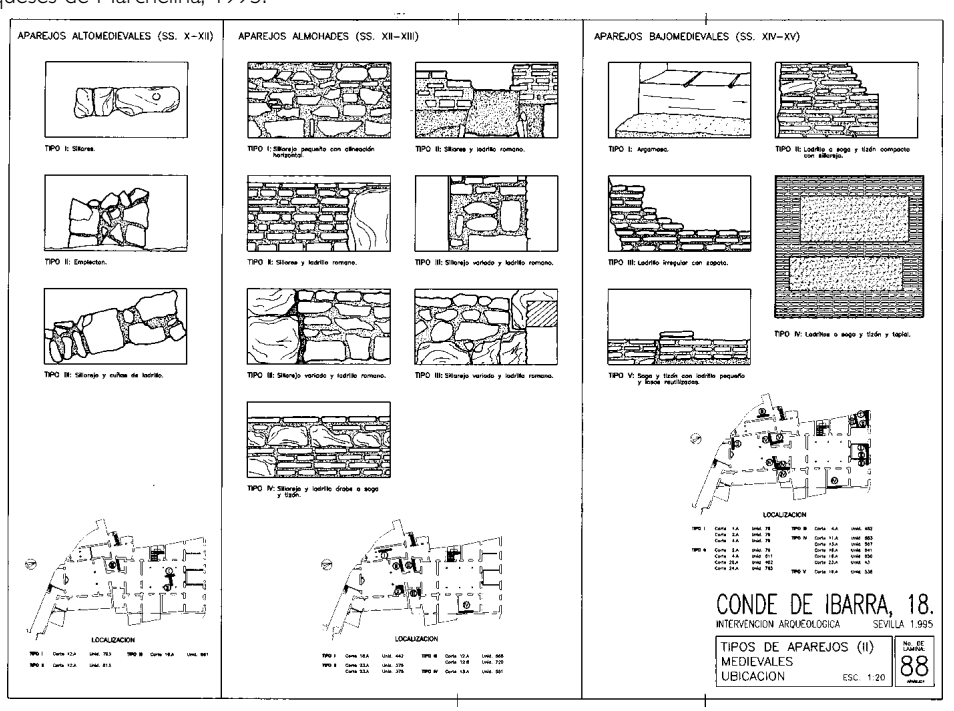

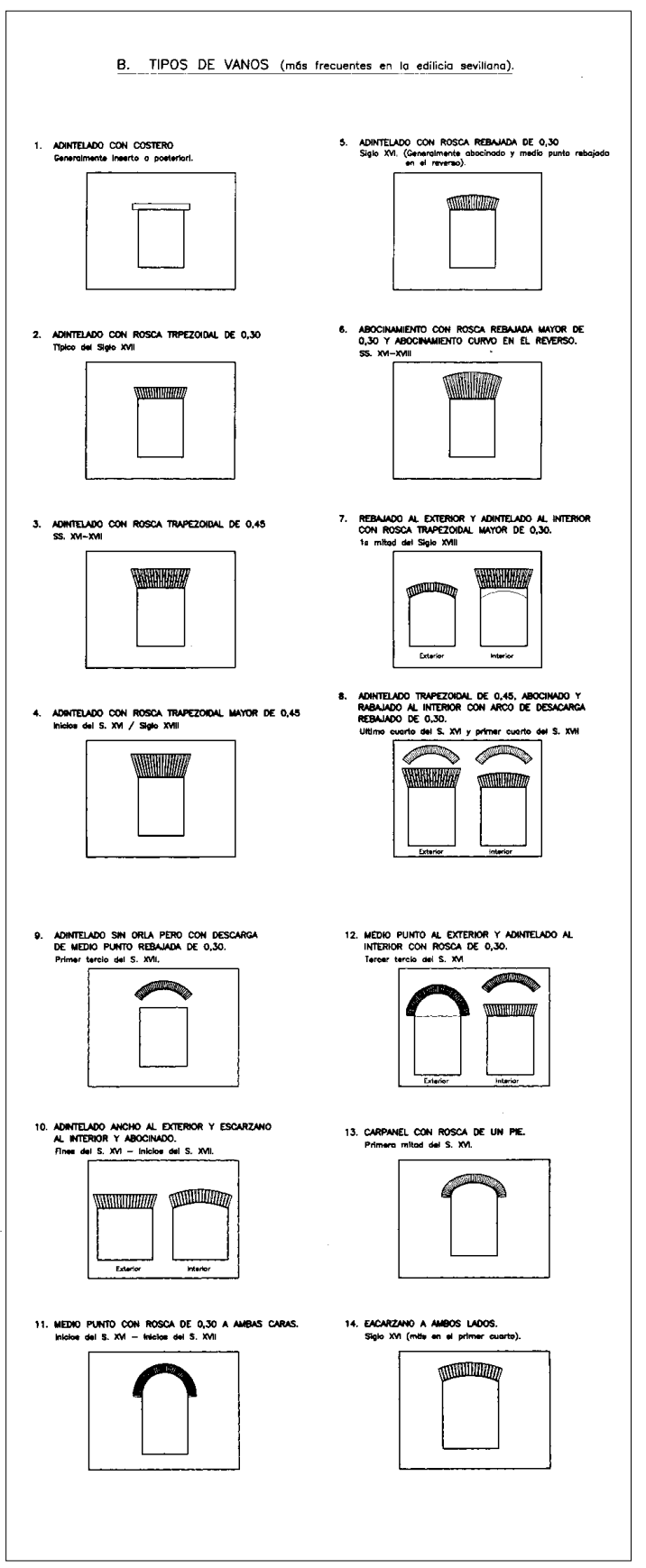

A continuación, y cuando los aspectos relacionados con la catalogación ya han sido superados, se acometen los estudios paramentales, divididos en dos enfoques diferentes, uno de tipo tipológico-estructural y otro evolutivo, en la totalidad de los paramentos o en una selección (en las zonas de especial interés).

Los análisis estratigráficos evolutivos de los paramentos siguen el sistema Harris simplificado en cuanto a las relaciones entre unidades, relacionando bloques de unidades con tramas aplicadas a una misma "fase" constructiva, no necesariamente cronológica. Para la comprensión evolutiva de la estructura se procederá al estudio minucioso de los alzados aún en pie. Este estudio se concentrará en aquellos paramentos en lo que se observen reformas claras o en aquellos que por su homogeneidad 
resulten básicos para el estudio global. El sistema empleado para este análisis paramental consiste en :

- Estrategia previa de picados de muros, llagueados o limpiezas de determinados elementos.

- Dibujo de cada paramento individualizado a escala |:50 preferentemente.

- Identificación del paramento mediante el número de estancia y su orientación cardinal, además de la referencia a la planta. Ejemplo: 27-Oeste, planta alta.

- Utilización de la ficha estratigráfica diseñada para el Cuartel del Carmen, especialmente transformada para incoporar mejor los datos de alzados.

- Dibujo exacto de los contornos de las unidades principales y simplificado de las secundarias. Esto es variable según el caso, tendiendose a la esquematización a medida que subimos la escala.

- Dibujo esquemático de los interiores de las unidades (fábricas murarias, rellenos, tapiados) salvo cuando presenten características particularmente interesantes, como en el caso de las reformas de las fábricas de muros.

- Utilización de tramas para cada época. Preferimos simplificar los eventos cronológicos en un número reducido de fases mucho mas comprensibles. Por supuesto esto es variable según el interés particular de la zona o el edificio pero debe procurarse no establecer mas de 5 ó 6 tramas cuando trabajamos a escalas 1:50 ó superiores.

- Numeración de las unidades principales, simplificando en un número aquellas que forman parte de un

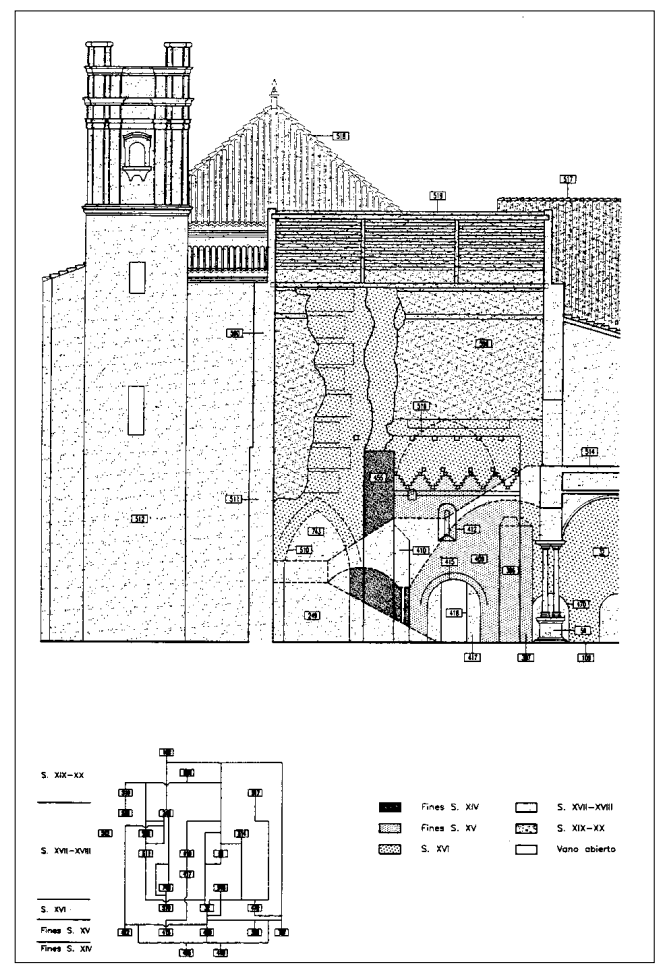

grupo homogéneo y coetáneo, como los mechinales de un forjado o las vigas de un techo. A este respecto la simplificación del método Harris, al igual que la excavación, nos permite concentrar nuestra labor de investigación en la globalidad del edificio. La aplicación exhaustiva de dicho método o de las versiones paramentales italianas de Parenti, Carandini, etc, sólo es posible con grandes equipos de profesionales concentados en pequeños edificios con pocas fases históricas de transformación.

Los estudios estructurales se dividen en dos (62).

I. Estructurales: Estableciendo mediante una simbología desarrollada por M.A.Tabales, (pero basada en Doglioni-1988) las relaciones físicas más importantes (rupturas, erosiones, adosamientos, grietas, etc...).

2. Tipológicos: Mediante un código creado al efecto, se identifican:

- Tipos de aparejo

- Tipos de vanos

- Tipos de enlucidos

- Tipos de añadidos

En el caso del aparejo, se subdivide en 4:

I. Ladrillo; con una subdivisión en tipos basadas en Clairac y Parenti, añadiendo tipos sevillanos identificados hasta el momento.

II. Piedra; siguiendo a Parenti, complementados por M.A. Tabales.

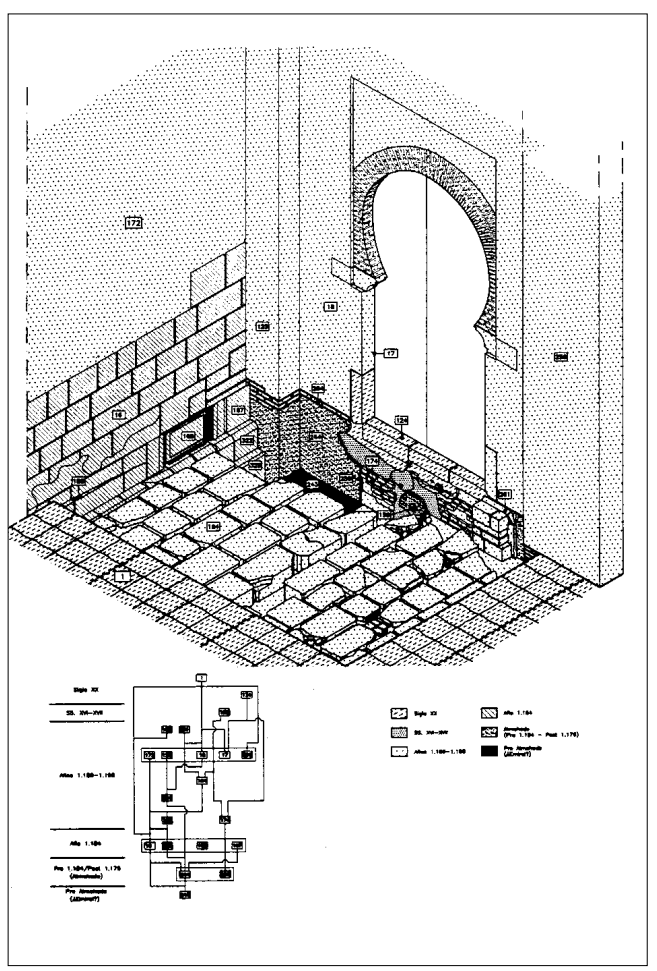

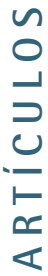

8. Análisis estratigráfico de paramentos. Ejemplo: Cuartel del Carmen, 1990.

9. Análisis estratigráfico de paramentos. Ejemplo: Giralda y Puerta del Lagarto (Catedral) 1996. 


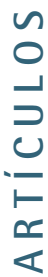

10. (sup.) Análisis tipológico y estructural de paramentos. Ejemplo Giralda y Puerta del Lagarto (Catedral), 1996

II. (inf.) Interpretación evolutiva final. Ejemplo: Iglesia de Santa Ana de Guadalcanal, 1996.

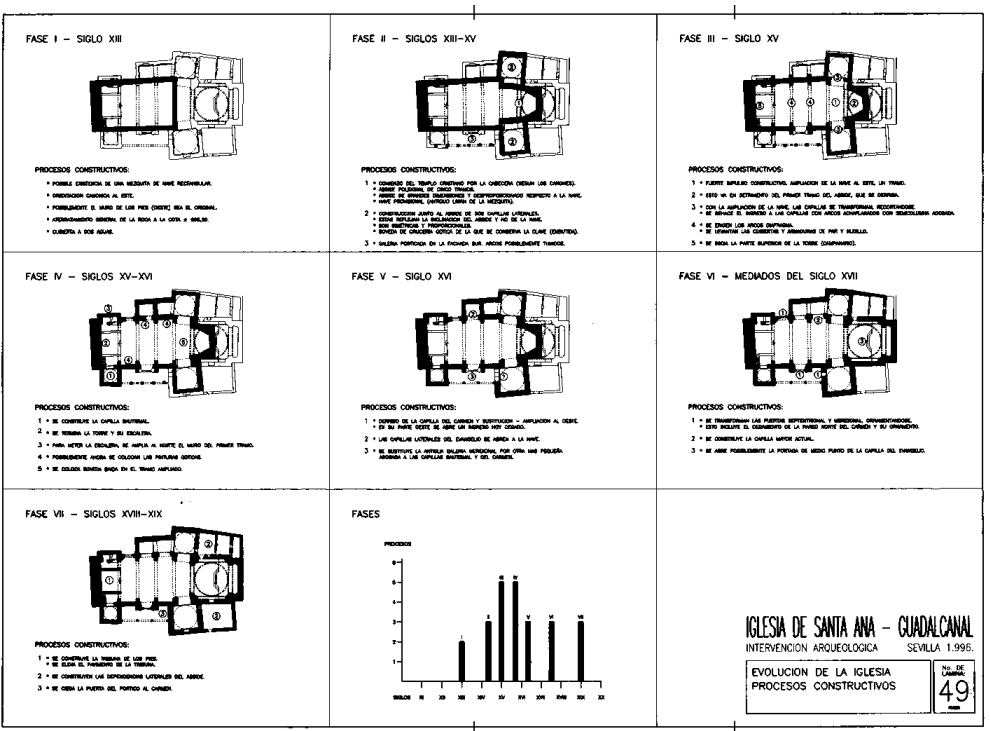

El estudio del subsuelo puede realizarse a la vez que el de los alzados, sin embargo en nuestra opinión es preferible su posterioridad, ya que existen muchos datos paramentales vinculados al subsuelo (cimientos, pavimentos, etc..) que deben conocerse antes de excavar con el fin de no desaprovechar el tiempo. Además, aunque es buena la especialización de uno de los miembros del equipo en las tareas de análisis de lo emergente, es beneficioso para la investigación que el mismo sujeto que ha estudiado los alzados de una zona, se encargue también de los trabajos de excavación.
Para el trabajo en general, será necesaria la división del edificio por estancias, numerándolas individualmente. Además se utilizará un punto "0", o cota referencial respecto a la ciudad.

De estos cortes, al menos uno será "estratigráfico", es decir, profundizando hasta lo posible y excavando con técnica arqueológica sistemática, analizando minuciosamente, todos los elementos del registro y edafológicos, sin selección alguna de materiales.

El resto de cortes arqueológicos serán de tipo zanja o cuadrícula, aunque cuando se considere necesaria una extracción de tierras (preparación para suelos, sótanos, cimentaciones, pozos, etc.) se procederá a una limpieza arqueológica previa o a un seguimiento exhaustivo a pie de obra.

El corte estratigráfico, se realizará en la zona menos peligrosa del edificio. El resto se distribuirá estratégicamente en la mayoría de las estancias, atendiendo, en principio a la búsqueda de los niveles guía (fases constructivas previas y cotas sucesivas), para lo cual se comenzará con la realización de cortes-sondeo o Cortes-Guía en areas dispersas y alejadas.

Los cortes restantes serán seleccionados a partir de ese momento y tendrán un carácter extensivo, es decir, no serán sistemáticos en cuanto a la recogida de elementos del registro, para concentrarse en el conocimiento en extensión de las fases previas.

La excavación, los análisis fotográficos de los sucesivos niveles, la plasmación gráfica de los resultados y la documentación sistemática, en algunos casos, y selectiva, en otros, se fundamentará en varios presupuestos básicos:

- Numeración de los cortes con la siguiente correlación: Estancia-Letra mayúscula del abecedario en orden de ejecución.

- Cota "0" homologada a la de la rehabilitación.

- Sistema de excavación selectivo y ágil en los análisis extensivos y en los rebajes zonales, pero sistemático en los sondeos y cortes estratigráficos.

- Aplicación del método "Harris" de documentación de unidades estratigráficas, simplificado en virtud de las necesidades concretas de la intervención. Ficha del Cuartel del Carmen(M.A. Tabales).

- Realización de filmaciones esporádicas de vídeo, así como fotografía y diapositivas de cada paso.

- Dibujo a diferentes escalas de cada nivel destacable y de los perfiles principales, con la escala 1:20 como base estandarizada estratigráfica.

Para poder llevar un control exhaustivo de los datos extraídos y los documentos generados por la investigación serán empleados varios registros: 
- Registro de unidades estratigráficas

- Registro de material gráfico

- Registro de bolsas

- Registro de cortes

- Registro de cajas

Cada uno de ellos llevará una ficha diferente y deberá estar cumplimentado al día. Además será necesario un control, igualmente cotidiano, de los materiales arqueológicos procedentes de cada corte. Para ello se procederá al lavado, siglado y descripción (en ficha especial) de cada pieza, así como su dibujo arqueológico, y su valoración cronológica. El fin de esta labor es el de disponer con agilidad de datos que podrán acelerar la valoración evolutiva de cada fase. Por tanto, uno de los miembros del equipo arqueológico debe estar concentrado a tiempo completo en estas operaciones de control.

Ya hemos comentado en cada caso el sistema de fichas diseñado por nuestro equipo. Se trata en primer lugar de la ficha de Unidades Estratigráficas, ideada para incorporar con la misma precisión que los datos estratigráficos los datos paramentales, mejorando así la plasmación de la tridimensionalidad de nuestro estudio; en esquema se caracteriza por:

- Una primera parte de identificación de la "unidad" dentro del corte, estancia y yacimiento.

- Le sigue un control de la disposición de la unidad, referenciada respecto de las que se relacionan directamente con ella, manifestándose su caracter de verticalidad, horizontalidad o interfacialidad.

- En cada uno de los tres casos posibles (capa, estructura o elemento interfacial) se han habilitado casilleros en los que se recogen las características más importantes. En el caso de que la unidad sea una capa, se refleja el origen, la deposición, la formación, la composición y la consistencia. En el caso de que la capa sea de tierra, se recoge su color y textura así como los componentes básicos. Por último, se refleja sintéticamente la proporción de materiales arqueológicos de la capa. En el caso de que sea una estructura, se incide en su caracter de verticalidad u horizontalidad así como en su ubicación estratigráfica, detallándose los materiales y técnicas empleadas para su erección, las dimensiones y un cuadro con observaciones. Si, por último, la unidad es una interfacie, se refleja su caracter respecto a las demás unidades y sus características esenciales.

- Se precisa una primera interpretación cronológica de la unidad, diferenciando claramente su fundamento absoluto o relativo.

- Respecto a los elementos documentales asociados a la unidad, quedan reflejadas las bolsas de materiales a través de su número de registro y su contenido, generalmente cerámica, restos oseos, vidrio, metal...).
- También se identifican los dibujos y planos en los que aparece la unidad así como los carretes de fotos, diapositivas o película de vídeo.

- Por último, se detalla, si se considera oportuno, algún aspecto general o concreto en un espacio cuadriculado para croquis, dejando un espacio para la plasmación de observaciones.

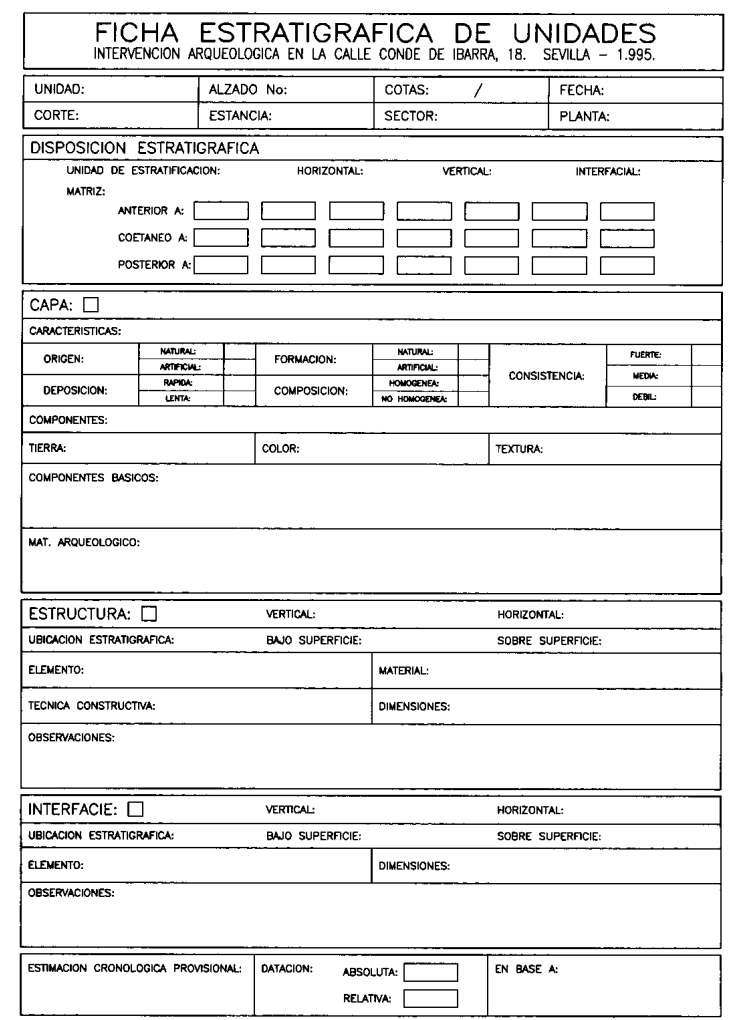

12. Ficha estratigráfica de Unidades. Anverso y reverso. Tabales, M.A. 1992. Sustituye a la experimentada en 1991 y 1992 en M. San Clemente y el Convento de S. M $M^{2}$ de los Reyes (ver bibliografía).

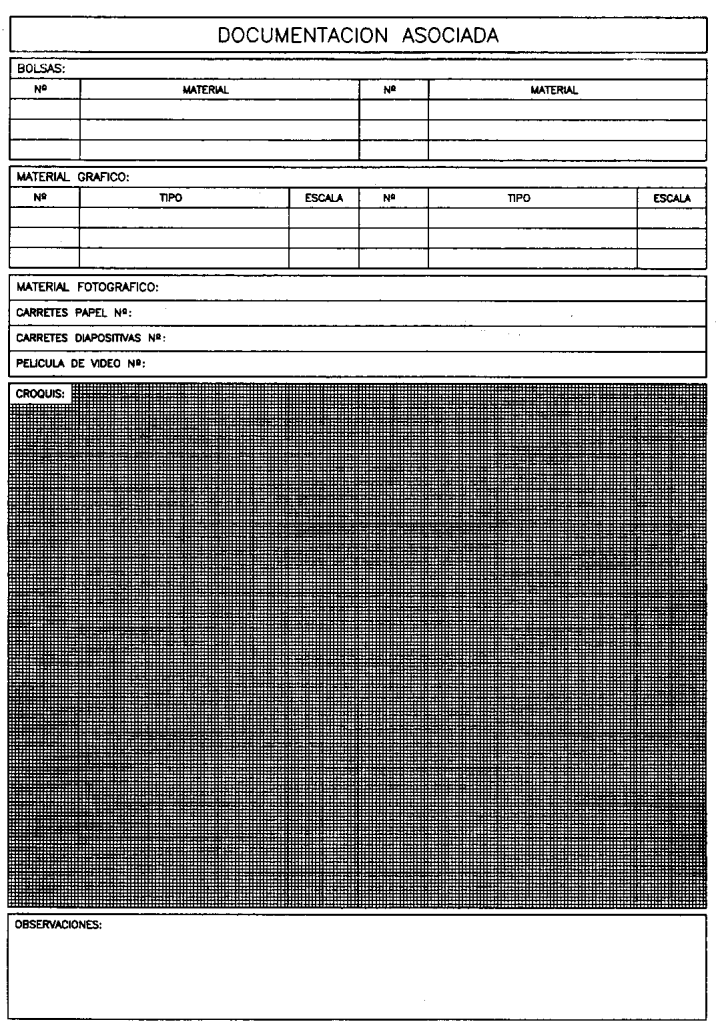




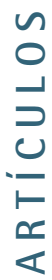

13. Ficha de Muestreo Edilicio. Tabales, M.A. 1994

14. Ficha de Control Tipológico. Tabales, M.A. 1994

I5. Ficha de Control Arqueológico Oliva, D. y Larrey E. 1989 y Tabales, 1990
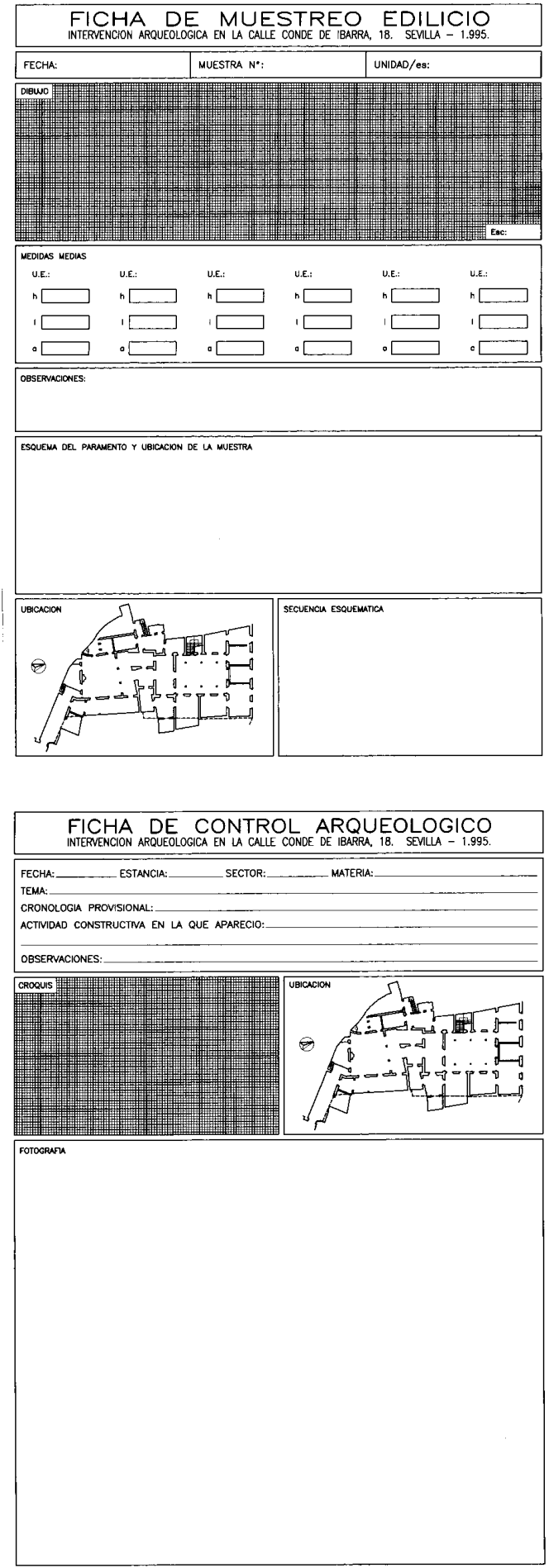

Del resto: la de control arqueológico, muestreos edilicios y control tipológico, ya se ha hablado anteriormente.

Todo el sistema, tanto en su vertiente estratégica, como en la de los registros, descansa sobre la aplicación del método Harris, con algunas puntualizaciones. Al referirse a un muro, Harris abstrae las relaciones para ajustar al método, de modo que lo imagina en disposición horizontal, dividiendo las unidades en unidades de estratificación vertical (muros, etc..), horizontal (enlucidos), unidades interfaciales de estrato vertical (superficies murarias), y unidades de estrato horizontales (grietas, superficies alteradas,

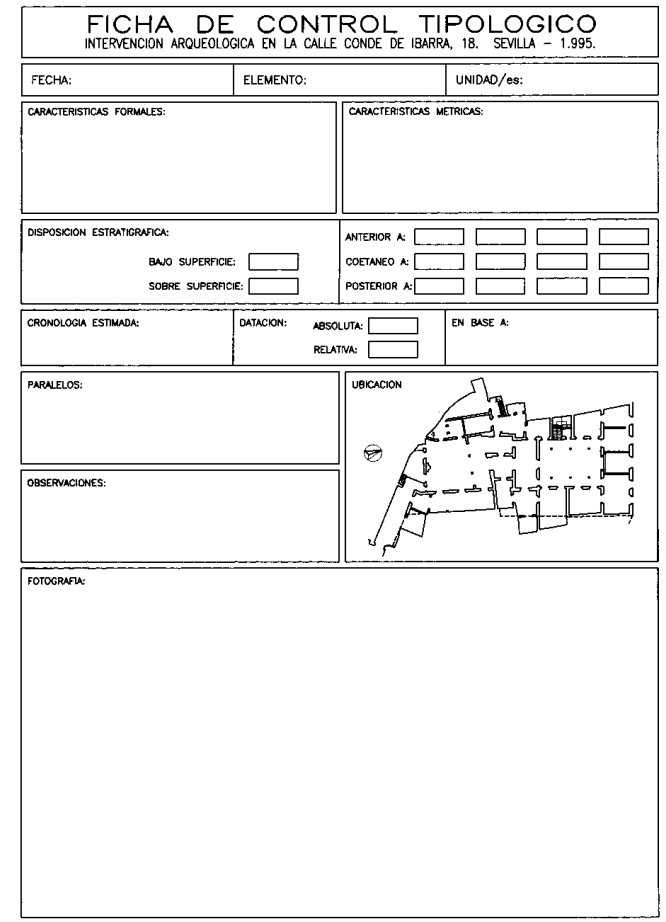

etc...) Por su parte Parenti extrae los muros del conjunto de la excavación y denomina a sus unidades, "unidades de estratificación muraria", dividiendo entre elementos estructurales, ornamentales, etc...

Por otro lado, Parenti crea relaciones distintas a parte de las tres de Harris (de anterioridad, coetaneidad y posterioridad), subdividiendo cada una segúl la relación (es cortado por, tangente a, se adosa, etc...) añadiendo gráficamente a la ley de contemporaneidad unalínea horizontal entre unidades coetáneas.

En definitiva, Harris intenta aplicar las relaciones y nomenclaturas estratigráficas a los muros, siendo el resultado necesariamente confuso, a poco que se analicen estructuras medianamente complejas.

Por su parte, Parenti desvincula lo emergente de lo soterrado, creando fichas distintas y simplificando mediante unas nomenclaturas nuevas, a la par que introduce la relación de contemporaneidad.

En nuestra opinión, la idea de Harris de no desvincular el sustrato de lo emergente es la correcta. De Parenti, sin embargo debe recogerse la idea de especificar las unidades paramentales, poco resueltas por Harris.

Así, en nuestro caso, la ficha estratigráfica es única y polivalente, pero recoge las características interfaciales y paramentales esenciales, no contemplando otras relaciones que las tres clásicas de Harris.

La característica esencial de nuestro sistema es la simplificación gradual de las unidades en función de nuestras necesidades; y ésto, que podría verse como algo prescindible, no es tal, si tenemos en cuenta la complejidad de los paramentos sevillanos, pudiendo convertirse, como de hecho sucedió en intervencio- 
nes ya citadas, en una rémora para la investigación global.

Desde la elaboración y práctica de este modelo hasta el presente, nuestro sistema se ha puesto en práctica en otros edificios como el Palacio de los Marqueses de Marchelina (63), la Iglesia de Santa Ana de Guadalcanal, la Puerta de Córdoba de Carmona (64), etc...extendiéndose entre otros profesionales sevillanos.
I. Tabales, M.A. Metodología arqueológica aplicada a la Rehabilitación de edificios. El Convento de Santa Maria de los Reyes de Sevilla. Tesis de Licenciatura, Universidad de Sevilla, Dpto. de Prehistoria y Arqueología, (inédito). Sevilla 1993.

2. Tabales, M.A. Arqueología y Rehabilitación de edificios históricos en Sevilla. Tesis Doctoral en curso. Prevista lectura 1997.

3. Como precursor en España de los estudios previos a la rehabilitación, Caballero Zoreda ha puesto en práctica sus ideas en múltiples intervenciones como las de Santa María de Melque, Santa Lucía del Trampal, etc..., destacando como aportaciones teóricas algunos artículos entre los que destacan por su caracter sintético: Caballero, L. "La intervención arquitectónica analizada desde la intervención arqueológica" en Actas del VII cursillo sobre la intervención en el patrimonio arquitectónico. Vic 1984. Barcelona, 1985. Otros investigadores como J.L. Latorre conforman este foco de investigación.

4. El Servicio de Conservación de la Diputación de Barcelona está dirigido por el arquitecto Antoni González, autor de múltiples ensayos sobre la metodología de la rehabilitación entre los que destacan: González, A. Recerca i disseny. El monument com a document historic i com objecte arquitectonic viu. Barcelona 1985; "A la recerca de la restauració objectiva" en Com i per a qui restaurem...Barcelona 1990; "Estudio, uso y abuso del patrimonio arquitectónico" en Monumentos y Proyecto. Jornadas sobre criterios de intervención en el patrimonio arquitectónico. Madrid 1990. etc.... entre otros.

Como responsable del área de investigación arqueológica, Albert López i Mullor, ha puesto en práctica el sistema de trabajo del Servicio, plasmando su experiencia metodológica en varios artículos entre los que destaca: López, A. "Consideraciones metodológicas sobre la actuación del Servicio de Catalogación y Conservación de Monumentos en el campo de la investigación arqueológica" en Actas del VII cursillo sobre la intervención en el patrimonuio arquitectónico,Vic 1984. Barcelona, 1984 ; o "Set anys d'investigació arqueológica del patrimoni arquitectonic" en Com i per a qui restaurem... Barcelona 1990.

5. En Sevilla se han puesto en práctica diversas experiencias en lo que respecta a la inserción de los estudios arqueológicos en el proceso de rehabilitación desde mediados de los años ochenta. A nivel metodológico destacan los trabajos de Diego Oliva Alonso en los Palacios de Altamira y Mañara entre los que destacan: Oliva, D. "La investigación en lo construido como apoyo a la restauración del Patrimonio Arquitectónico" en Restauración Casa-palacio de Miguel de Mañara, Sevilla 1993.; o los realizados por M.A. Tabales Metodología arqueológica aplicada a la rehabilitación de edificios. El Convento de Santa María de los Reyes de Sevilla. Tesis de Licenciatura, Sevilla 1992.(inédita); "Aspectos metodológicos" en Intervención arqueológica en el Real Monasterio de San Clemente de Sevilla, (en prensa).
La experiencia en la Cartuja y en otros edificios sevillanos hacen destacar la labor pionera de Don Fernando Amores, verdadero impulsor junto a D. Oliva de este tipo de análisis en nuestra ciudad.

6. Harris, Edward C. Principios de estratigrafía arqueológica. Barcelona 1991. (traducción del original inglés, Londres 1979).

7. Carandini, Andrea Storie dalla Terra. Manuale dello scavo archeologico. Bari 1981.

8. Tagliabue, Rita, Architetto e archeologo. Confronto fra campi disciplinari. Milano 1993.

9. Brogiolo, Gian Pietro, Apunti ed imagini per l'analisi stratigrafica dell'edilizia storica. 1987; Archeologia dell'edilizia storica, Como 1988; "Campionatura e obiettivi nell'analisi stratigrafica degli elevati" en Archeologia e restauro dei Monumenti. Firenze 1988.

10. Roberto Parenti ha publicado una gran cantidad de estudios sobre arqueología paramental, destacando: Parenti, R. "Le strutture murarie: problemi di metodo e prospettive di recerca. El progetto Montarrenti (siena)", en Archeologia Medievale XII. Firenze 1985; "La tecniche di documentazione per una lettura strattigrafica dell'elevato" en Archeologia e Restauro dei Monumenti. Firenze 1988., etc...

II. Ricardo Francovich ha sido uno de los padres de la arqueología de Aoyo ala Restauración.. En su haber constan una gran cantidad de obras sobre metodología desde los años setenta, destacando: Francovich,R. "Alcuni problemi dei rapporti pratici fra archeologia, Restauro e Pianificazione Territoriale" en Archeologia Medievale VI, Firenze 1979.; "Restauro architettonico e Archeologia stratificata" Contributi sul restauro archeologico, Firenze 1982. Junto a Roberto Parenti dirigió el compendio: Archeologia e Restauro dei Monumenti. Firenze 1988.

12. Barker, Philip. "Tecniche dello scavo archeologico" a cura di Bruno d'Agostino) Milán 1981.

\section{Harris,E.C. Opus cit}

14. Carver, Martin "Valutacione, strategia ed analisi nei siti pluristartificati" en Archeologia Medievale X, Firenze 1983.

15. Hurst, H. "La stratigarfia degli elevati" en Atti del Convegno Come L'archeologo opera sul campo, Siena 1981.

16. Rahtz, P.H. "Archaeologists and architects: Areas of communication, collaboration and conflict" en Archaeological papers from York.York, 1984.

17. Davies, M. "The Archaeology of standing structures" en Australian Journal of Historical Archaeology, 5, 1987. 
18."Westfalen. Hefte fur geschichte Kunst und volkskunde".V.1977,3-4, DenKmalpflege in Westfalen-Lippe 1972-1976. Bauforschung und Grabungen.

19. Maetzke,G., Rysiewska,T., Tabaczynski,S., Urbanczyk,P. "Problemi dell'analisi descrittiva nelle ricerche sui sitti archeologici pluristratificati" en Archeologia Medievale IV, Firenze 1977.

20. Lauffray,J. "Role de I'Architecture sur le chantiers de fuilles archèologiques. Ses rapports avec I'Archeologue" en II monumento per l'uomo. Atti del II Congresso Internazionale del Restauro, Maggio 1964, ICOMOS, Bologna 1972. Sanpaolesi, P. "Etudes preliminaires" en La conservation et la restauration des monuments et des bâtiments histoiques. UNESCO, Paris 1973.

21. De Bouard, M. "Storia e Archeologia nello studio dei Castelli medievali", en Castelli, storia e archeologia. Torino 198I.

22. Carandini, A. "Per una carta dello scavo archeologico" en Archeologia Medievale VI, Firenze 1977; Storie dalla Terra. Manuale dello scavo archeologico. Bari 1981.; "Urbanística, Architettura e Archeologia" en Urbanística,88, Milano 1987; "Archeologi e Architetti-Restauratori a confronto: alcune reflessioni di carattere generale" en Archeologia e Restauro dei Monumenti, Firenze 1988.

Carandini, A. y Pucci G. "L'Archeologiaoggi", La difessa del patrimonio artistico, Testi per Italia Nostra. Milano 1978.

23. Francovich,R. "Alcuni problemi dei rapporti pratici fra Archeologia, Restauro e Pianificazione Territoriale", Archeologia Medievale, VI, Firenze 1979.

24. Gurrieri,F. "Architetto, Archeologo, Centro Storico" en Archeologia Medievale, VI, Firenze 1979.

25. Bonora,F. "Nota su un archeologia dell'edilizia" en Archeologia Medievale,VI, Firenze 1979.

26. Mannoni, T. "Metodi di datazioni dell'edilizia storica" en Archeologia Medievale XI, Firenze 1984.

27. Fossati,S. "La datazione dei matoni: una proposta di metodo" en Archeologia Medievale XII, Firenze 1985.

28. Ferrando, I. "Tecniche díndagine per un'archeologia dell'edilizia povera" en Archeologia Medievale, VIII, Firenze 198I.

29. Parenti,R. "Le strutture murarie: problemi di metodo e prospettive di ricerca" en II Progetto Montarrenti (Siena), en Archeologia Medievale XII, Firenze 1985.; también "La torre A: una lettura stratigrafica" en II progetto Montarrenti (Siena), en Archeologia Medievale XII, Firenze 1985.; y “La lettura stratigrafica delle murature in contesti archeologici e di restauro architettonico", en Restauro e Città, 2, 1985.

30. Bonelli, R "Archeologia stratificata e Storia dell'Architettura" en Architettura. Storia e documenti, 2, 1986.

31. Francovich,R. y Parenti,R., (a cura di) Archeologia e Restauro dei Monumenti. Firenze 1988.

32. Ferrando,l. "Archeologia e conservazione: L'esperienza ligure" en Archeologia e Restauro dei Monumenti, Firenze 1988. Manacorda,D. "Apunti su Archeologia e Architettura nel can- tiere della Crypta Balbi" en Archaeologia e Storiadel Medioevo italiano (a cura di R. Francovich), Roma 1987.

33. Marino,L. "Archeologia e Restauro. Restauro dell'Archeologia" en Archeologia e Restauro dei Monumenti. Firenze 1988.

34. Doglioni,F. "La ricerca sulle strutture edilizie tra aecheologia stratigrafica e restauro architettonico". Archeologia e Restauro dei Monumenti. Firenze 1988.

35. Parenti,R. "La tecniche di documentazione per una lettura stratigrafica dell'elevato". en Archeologia e Restauro dei Monumenti. Firenze 1988

36. Medri, M. "La pianta composita nella documentazione e nell 'interpretazione dello scavo" en Archeologia e Restauro dei Monumenti, Firenze 1988.

37. Brogiolo,G.P. Archeologia de la edilizia storica, como 1988; también "Campionatura e obietivi nell'analisi stratigrafica degli elevati"' en Archeologia e Restauro dei Monumenti, Firenze 1988.

38. Tagliabue,R. Architetto e Archeologo. Confronto fra campi disciplinari. Milano 1993.

39. Somos conscientes de que, por lo delicado del tema, las soluciones futuras, ya sean asumidas mediante una legislación adecuada, o bien mediante una actitud de facto, nunca satisfacerán completamente a todos los profesionales implicados, ya que en el transfondo no se encuentra una actitud de búsqueda objetiva de soluciones ideales en la rehabilitación, sino una lucha de intereses que impregna a cada colectivo (historiadores, arqueólogos, arquitectos...). Existe una mentalidad forjada desde las mismas facultades y escuelas, basadas en la idea de superioridad y de supeditación de cada profesión; y esta actitud se transluce a la más mínima ocasión. Un arqueólogo especializado aceptará con dificultad la innata pretensión del arquitecto por dejar su huella en el edificio, y en el fondo siempre aspirará a participar en las decisiones importantes; por su parte un historiador del Arte jamás aceptará internamente la supeditación a un equipo dirigido por arqueólogos, aunque sí a un arquitecto, y por otro lado, ningún arquitecto renunciará su capacidad decisoria final, y menos ante profesionales que no sólo coartan su capacidad de diseñar, sino que poco a poco van asumiendo actividades tradicionalmente impulsadas por ellos.

40. Colegio Oficial de Arquitectos de Andalucía Occidental.

41. Parenti y Francovich, opus cit, Florencia 1988.

42. F. Amores desempeñó esta tarea de 1985 a 1986.

43. J. M. Campos fue arqueólogo provincial de 1986 a 1988.

44. En el caso de la Hacienda de Miraflores, catalogada y prospectada por F. Amores y J.M. Campos, en 1985, se documentó todo el proceso de ocupación humana, ampliándose con el descubrimiento de una villa romana, el conjunto islámico ya conocido. Además, se realizó una investigación documental sobre textos medievales relativos a los sistemas hidráulicos constatados. Las excavaciones comenzaron al año siguiente a cargo de C. Romero, quien documentó la torre almohade de la alquería y realizó excavaciones en el sitema de regadío. 
45. Junto a F. Mendoza Castells, F. Amores y J.M. Campos procedieron a la catalogación, revisión y calificación urbanística de las huertas del palacio almohade del siglo XII y sus huertas.

46. En el caso de las Murallas de la Macarena, en 1985, J.M.Campos y M. Teresa Moreno, realizaron una investigación documental, toma de cotas y referencias tipológicas de la construcción, que obligaron a la transformación del proyecto original, constituyéndose ésta como la primera intervención real de apoyo a la restauración en Sevilla.

47. J.M. Campos y J. Escudero "Intervención en el antiguo Noviciado de San Luis...." en A.A.A. 1986. Sevilla 1987.

48. Todos ellos publicados en el A.A.A. de 1986, Sevilla, 1987, a cargo de: C. Romero (Miraflores), J. Campos y J. Escudero ( San Agustín); Campos, Amores y M. Vera (Cartuja), M.T. Moreno y M. Vera (Murallas del Valle) y J. Campos y M. Vera y M,T. Moreno ( La casa de la Moneda).

49. En principio dirigidas por F. Amores y J. M. Campos y finalmente por el primero.

50. Los trabajos en el noviciado de San Luis durante 1987 fueron dirigidos por J.Escudero y en 1988 por J. Campos y A. Gómez. Véanse Anuarios Arqueológicos de Andalucía correspondientes a ambos años.

51. La intervención en el Palacio de Altamira fue dirigida por E. Larrey y D. Oliva, siendo Francisco Torres arquitecto.

52. En 1989 se llevó a cabo una primera campaña de aproximación al Palacio de Mañara a cargo de S. Rodríguez y D. Oliva. Más adelante, durante todo el año 1990 se llevaría a cabo uno de los análisis más completos realizados hasta ahora en nuestra ciudad, a cargo de D. Oliva, R. Ojeda y M.A. Tabales; véanse Anuarios Arqueológicos de Andalucía de los años 1989 y 1990. En el caso del Palacio de Mañara, Varios Autores, Rehabilitación casa de Miguel Mañara. Sevilla 1993; así como Ojeda/Tabales, “Estudio diacrónico.....'IV Jornadas de Arqueología Medieval, Alicante 1993, etc...

53. En el año 1989 se realizó una primera campaña en el Monasterio de San Clemente a cargo de R. Ojeda. Durante el año 199| se acometió el análisis integral del inmueble (M.A. Tabales) Intervención arqueológica en el Real Monasterio de San Clemente, Sevilla. A.A.A. Sevilla, I.991.

54. Trabajo dirigido por F. Pozo y M.A. Tabales en 1988, y por F. Pozo en 1990. Intervención arqueológica en el Monasterio de San Jerónimo. Anuario Arqueológico Andaluz. SeviIla, 1.989.

55. Trabajos dirigidos por la Profesora M. Valor y por N. Casquete.
56. Trabajos dirigidos por M.A. Tabales con la finalidad de desarrollar la presente investigación metodológica. Junto a dicho investigador, codirigieron en el caso del Cuartel del Carmen, Intervención arqueológica en el Cuartel del Carmen de SeviIla, A.A.A. Sevilla, 1.993, y el Palacio de los marqueses de Marchelina, D. F. Pozo y D. Oliva. En el caso de la Puerta de Córdoba, dirigió D. Reyes Ojeda, quien desde el IAPH, aplicó su sistema de registro, generado para el IAPH, junto a nuestro modelo de análisis y estrategia de actuación.

57. Trabajos dirigidos por M. A. Tabales, F. Pozo y D. Oliva.

58. Pozo ,F. Y Tabales, M.A. Intervención arqueológica de apoyo a la restauración en el Castillo de Alcalá de Guadaira. Anuario Arqueológico Andaluz. Sevilla, 1.989.

59. Oliva, Ojeda y Tabales:"Intervención arqueológica de apoyo a la restauración del Palacio de Mañara, Sevilla. A.A.A. Sevilla, 1.990; "Intervención arqueológica en el Palacio de Mañara". Actas de las IV Jornadas de Arqueología andaluza" ( resúmenes). Jaén, 1991; Tabales,M. y Ojeda,R. "El muro de la judería sevillana: su recuperación en la casa de Mañara", en La casa Palacio de don Miguel de Mañara, Sevilla, 1992; Tabales, M.A. "La casa mudéjar", en "La Casa-Palacio de Don Miguel de Mañara, Sevilla, 1.992;Ojeda, R. Tabales, M.A. "Estudio discrónico de la ocupación del edificio islamico ubicado bajo la Casa de Mañara (Sevilla), tres usos, tres culturas (ss.XII-XV)." Actas del IV Congreso de Arqueología Medieval Española, Alicante, 1994.

60. Tabales, M.A. "El Monasterio de San Clemente. Superposición cisterciense sobre un edificio almohade".'Actas del IV Congreso de Arqueología medieval española",Alicante, 1994; "El edificio musulmán excavado bajo el Convento de San Clemente de Sevilla". Catálogo exposición "El último siglo de la Sevilla islámica", dirigida por la Dra. Magdalena Valor Piechotta. Sevilla 1995; Intervención arqueológica en el Real Monasterio de San Clemente. Sevilla, 1.992. (en prensa).Ed. Fundación el Monte.

61. Tabales, M.A. "Intervención arqueológica en el Convento de Santa María de los Reyes de Sevilla", A.A.A. Sevilla, I.992.

62. Las distintas tipologías (aparejos, tipos de vanos, etc...) organizadas por el autor, han sido utilizadas de manera provisional en varios edificios sevillanos, y verán la luz en breve en la citada Tesis Doctoral.

63. Tabales, M.A., Pozo, F. y Oliva, D.'Intervención arqueológica en Conde de Ibarra 18. El edificio almohade" en Catálogo exposición "El último siglo de la Sevilla islámica", dirigida por la Dra. Magdalena Valor Piechotta. Sevilla 1995.

64. Ojeda, R. y Tabales, M.A. "La investigación arqueológica en Bienes Inmuebles. Metodología aplicada en la Puerta de Córdoba de Carmona", en Boletín del IAPH n I5, Sevilla, Junio de 1996. 